\title{
Efficient health research and improving Europeans' health
}

\author{
Fernando Antoñanzas ${ }^{1}$ Carmelo A. Juárez-Castelló ${ }^{1} \cdot$ Roberto Rodríguez-Ibeas $^{1}$
}

Received: 5 May 2015/Accepted: 8 May 2015/Published online: 24 May 2015

(C) Springer-Verlag Berlin Heidelberg 2015

The EU Framework Programme for Research and Innovation (FP) Horizon 2020, for the period 2014-2020, represents a significant effort to support research activities. Almost 80 billion euros in current prices are going to be allocated to these activities over 7 years (versus the 50 billion budget of the 7th FP) which, under three major headings_Excellent Science, Industrial Leadership and Societal Challenges - have recently opened their calls for research projects. Health and biomedical research initiatives can be mostly channeled through the "Health, demographic change and wellbeing" chapter of the Societal Challenges heading, representing about $10 \%$ of the total budget [1]. Furthermore, some other health related projects could also apply for funds under the "Food Security" and the "European Research Council" chapters, with the latter falling into the Excellent Science heading. In comparative terms with the last EU 7th FP, Horizon 2020 has notably increased the budget specifically dedicated to health research (from $€ 5.57$ up to $€ 8.03$ billion). In addition to this FP, the EU is currently running the 3rd Health Programme [2]; it is considered to be the main instrument for implementing the EU Health Strategy (funded with about $€ 450$ million) and is complementary to the EU FP Horizon 2020. Among other goals, this programme specifically pursues research into public health, the efficiency and sustainability of health systems and human resources management.

When such an important amount of public resources is spent (i.e. invested) to finance research, evaluation activities, ex ante and ex post, are crucial in the whole

Fernando Antoñanzas

fernando.antonanzas@unirioja.es

1 Department of Economics, University of La Rioja, Logroño, Spain process. After identifying the potential research topics, a first evaluation (qualitative at this stage) must be performed to prioritize them under different criteria (health improvements for some target populations, integration of research networks, profitability for the industry, potential stakeholders for the innovation, and so on). The targets of the calls of the FPs usually reflect the interests of several stakeholders, represented by the Advisory Groups (AGs), the European Technology Platforms (ETPs) and the Programme Committees (PCs). The specific work-programmes are elaborated and reviewed by these three groups, with representatives of Member States in the PCs.

Then, the call for proposals is issued and a second evaluation has to be carried out to select which ones are funded. The purpose of this evaluation is to reduce the uncertainties of allocating resources to some groups that may not perform the research as desired, and rather assign funds to those groups more likely to achieve the expected results. This evaluation considers several aspects, such the adequacy of the team and its critical mass for the projects, the fragmentation/integration of the research, the experience of the research team, the adequacy of means and planned activities of the project, the legality of the procedures, etc. This evaluation is carried out by panels of independent experts, organized by and under the supervision of the Directorate General Research and Innovation (DG R\&I), and enhances the efficiency of the research process, although it does not directly perform an economic evaluation: that would be very difficult at this stage.

Another evaluation-ex post-is implemented after the execution of the research process. Although this evaluation should theoretically assess the value of the research outcomes, in practical terms it mainly focuses on administrative aspects (control of invoices, payments, and the period between the authorization to run the research and 
the starting moment) and other intermediate endpoints of the research activities, such as the number of publications and presentations in scientific meetings. If the publicly funded research activities must be efficient, it would be necessary to include an evaluation from the economic perspective. However, this task is not straightforward given the intangibility of some results of the projects, the long process from target exploration to implementation in biomedical and heath research and the difficulties of identifying whether future developments were based on the results of some previous projects. This is also related to a lack of follow-up information as to whether the implementation of some health policies really derived from the project results. These issues are even more complicated to cope with in the field of health due to the influence of other confounding variables. Therefore, the estimation of the efficiency of the research has not been directly performed.

The EU has several Joint Research Centres, involving several groups of experts who directly develop some research for the European Commission and support the evaluation of the FP projects. In the year 2008, a group of these experts presented the evaluation of the 6th FP (2002-2006) and also an interim report in 2010 of the 7 th FP (the final report is still under elaboration) [3]. From these reports some lessons, mostly related to the administrative management of the processes of the calls, are useful for improving the elaboration of future FPs, out of a general belief that these programs contribute very much to the promotion of EU research and collaboration of international teams. Anyway, these Centres do not assess the efficiency of FP results as such.

Although developing high quality results is a common goal in the research community, the final results do not always respond to the initial expectations. In economic terms, not all research is efficient in terms of achieving new knowledge, utility for the companies, and greater health and wellbeing for the citizens. The issue here is that if the major and general goal is to improve health, it can directly be achieved through several means: (1) specific research targeting some identified needs (such as those related to public health, health promotion and some types of illness prevention, health systems organization and disease management policies-guidelines, protocols, etc.-); interestingly, this research will not yield any patentable results and will automatically become public knowledge that could be implemented by any health system; (2) research that will need the partnership of the industry and, probably, generate some patents of new products (such as the development of a new drug); and (3) basic research to better understand human biology as an initial step in improving future health (again, the results of this kind of research will be accessible once published in specialized journals, and could be directly appropriated by different stakeholders to develop health technologies in the future).
Therefore, the estimation of the efficiency of such quite different types of research is cumbersome as the time lags between the knowledge generation and the transmission of the innovations to society and, consequently, health improvements, may take several years. Furthermore, not all new knowledge generates a new technology. Also, even for new technology sometimes there is no market, or it is rather limited; sometimes new knowledge allows a new approach to the disease but further research establishes improvement over the existing treatments, and so on. That is to say, biomedical and health research needs a powerful "transmission belt" to reach the final target of health improvement, and there is an element of chance-no guaranteed success. Different interests have to be aligned as a necessary condition for the research to hit the final target and be brought to the market. Even then, the progress from market to patients has hurdles. Health policies need citizens' acceptance and participation as well. Otherwise, research outcomes may be delayed before being applied and taking effect. For instance, there is a significant amount of knowledge on several public health issues (such as the consequences of tobacco and alcohol consumption together with sedentary lifestyles) that only needs to be cleverly implemented together with peoples willingness to modify their behavior; these public health policies can generate health improvement beyond other medical interventions based on complicated research and development processes; however, the reinforcement is complex and slow.

So far, the list of topics in the health area prioritized under the Horizon 2020 mostly deal with the current health challenges of the developed world (few topics targeting public health issues) such as degenerative diseases, chronic conditions, aging, and aim for advanced approaches such as though personalized medicine, genomic knowledge, and regenerative technologies. Other research efforts are promoted in the area of rare diseases and in the design of vaccines useful for some diseases mostly prevalent in less developed countries, such as malaria. However, in spite of the importance of the role of health resources and process management as well as health systems organization, the scope for research on these aspects of health implementation in the current calls is quite limited. These three topics are more directly addressed within the frame of the EU Health Programme and administered under DG Health and Food safety, where there is a clear possibility of applying for funds to analyze the current situation of their structure, incentives and ways of improving the efficiency. In fact, perhaps as a consequence of these limitations, it was acknowledged somewhere else [4] that there is a clear shortage of publications on these topics, and thus the related policies may have been adopted without a clear understanding of their impact on both the productivity of the systems and citizens'health. In general terms, we can state 
that economics or health economics are scarcely used in defining the topics for new projects, policies or managerial issues that could promote health improvements.

Regarding the efficiency criteria in guiding research, it will be very difficult to end up with a quantitative scale as a tool for discriminating among project proposals. However, if applied on a rather qualitative basis, they could orientate the research towards those fields where the results were more easily translated into valuable health outcomes. This is a major challenge for those who must adopt the decisions on topic selection and on the a priori assessment of the proposals.

A novel approach for improving the processes in setting the global research agenda was included into Horizon 2020, strongly supported by the EU Parliament, who is concerned with the necessity of improving health research as a warrant for future health for EU citizens. A Scientific Panel for Health (SPH) has been set up by DG R\&I and its Health Directorate, and is composed of experts across different disciplines and areas [5]. Its mandate as set out in the terms of reference are: (1) to analyze bottlenecks preventing the achievement of better health and wellbeing and to propose solutions related to science, research and innovation to address these bottlenecks in the medium and longer term, (2) to identify long-term trends influencing health through recommended research, and innovation to respond to them, and (3) to propose strategies to promote the effective exploitation of knowledge generated at national and EU levels by research and innovation programs through the translation and implementation of results into practice. These terms aim to channel research efforts to future health challenges and to create the necessary environment so that research outputs become implemented by the health systems and end up having a practical utility.

Some examples of bottlenecks can be within the regulatory framework. There is a risk that revision of the EU data protection regulation would make the integration of data from different sources and their joint analysis more difficult. Another example is the regulation related to the approval of clinical trials, which currently requires assessment of the dossiers by several ethical committees, a process that easily generates delays in the generation of results and increases research costs. By addressing regulatory barriers, a greater efficiency in research and its implementation could be achieved. Having a longer-term foresight could help to set strategic priorities which build on recent developments and achievements, and could strengthen research. Finally, examining and addressing hurdles in the translation of results into practice can include the interaction and models for collaboration from discovery to implementation (i.e. organization of health care systems, economic incentives of health care providers to develop and implement the initial and original research outcomes, and so on). None of these terms is trivial at all; they are probably not too costly in budgetary terms, but they definitively require good doses of political interest, organizational changes and citizens' participation.

Another good example of an EU challenge!

Acknowledgments The authors would like to thank Prof. Karin Sipido (director of the Scientific Panel for Health) for her useful comments and suggestions. The usual disclaimer applies.

\section{References}

1. http://ec.europa.eu/research/horizon2020/pdf/press/horizon_2020_ budget_constant_2011.pdf. Accessed 10 Mar 2015

2. http://ec.europa.eu/health/programme/policy/index_en.html. Accessed 10 Mar 2015

3. http://ec.europa.eu/research/evaluations/pdf/archive/other_reports_ studies_and_documents/fp7_interim_evaluation_expert_group_ report.pdf. Accessed 10 Mar 2015

4. Antoñanzas, F., Rodríguez-Ibeas, R.: Channeling health economics research initiatives to improve decision-making processes in the EU. Eur J Health Econ 14(6), 847-852 (2013)

5. https://ec.europa.eu/programmes/horizon2020/en/h2020-section/ scientific-panel-health-sph. Accessed 10 Mar 2015 